\title{
An Efficient Fault Tolerant Cloud Market Mechanism for Profit Maximization
}

\author{
Boyu Li, Guanquan Xu, Bin Wu, Yuhan Dong \\ Tianjin University \\ Tianjin, China \\ \{boyulee,losin,binw\}@tju.edu.cn,Phoebe_Dong1@outlook.com
}

\begin{abstract}
In support of effectively discovering the market value of resources and dynamic resource provisioning, auction design has recently been studied in the cloud. However, there are limitations due to the inability to accept time-varying user demands or offline settings. These limitations create a large gap between the real needs of users and the services available from cloud providers. In addition, existing auction mechanisms do not consider service interruption due to server failures caused by software or hardware problems. To address the limitations of existing auction mechanisms and to avoid service interruption, this paper targets a more general scenario of online cloud resource auction design where: 1) users can request multiple types of time-varying resources; and 2) at least one server is available for each accepted bid even when one or more servers fail; and 3) profit is maximized over the system execution span. Specifically, we model the profit maximization problem using an Integral Linear Programming (ILP) optimization framework, which offers an elastic model for time-varying user demands. In addition, we design an online, truthful, and time efficient auction mechanism consisting of a price-based allocation strategy and a pricing function. The online allocation strategy allocates multiple types of resource to each user while satisfying the time-varying demands and ensuring at least one server is available for each user in each allocated time slot. Lastly, the efficacy of online auctions is validated through careful theoretical analysis and trace-driven simulation studies.
\end{abstract}

\section{CCS CONCEPTS}

- Theory of computation $\rightarrow$ Online algorithms.

\section{KEYWORDS}

cloud computing, auction, resource allocation,pricing, online algorithm, truthful mechanisms

ACM Reference Format:

Boyu Li, Guanquan Xu, Bin Wu, Yuhan Dong. 2021. An Efficient Fault Tolerant Cloud Market Mechanism for Profit Maximization. In Computing Frontiers Conference (CF '21), May 11-13, 2021, Virtual Conference, Italy. ACM, New York, NY, USA, 9 pages. https://doi.org/10.1145/3457388.3458669

Permission to make digital or hard copies of all or part of this work for personal or classroom use is granted without fee provided that copies are not made or distributed for profit or commercial advantage and that copies bear this notice and the full citation on the first page. Copyrights for components of this work owned by others than ACM must be honored. Abstracting with credit is permitted. To copy otherwise, or republish, to post on servers or to redistribute to lists, requires prior specific permission and/or a fee. Request permissions from permissions@acm.org.

CF '21, May 11-13, 2021, Virtual Conference, Italy

(C) 2021 Association for Computing Machinery.

ACM ISBN 978-1-4503-8404-9/21/05 . .\$15.00

https://doi.org/10.1145/3457388.3458669

\section{INTRODUCTION}

In cloud computing, a resource provisioning mechanism is often required to assign users a set of computing resources for task processing and data storage [3]. The elastic and on-demand nature of cloud computing enables users to meet their resource demands with minimal management overhead, while the cloud ecosystem as a whole achieves economies of scale through cost amortization. Currently, most cloud providers adopt a fixed-price policy to charge users for using pre-defined resource units. For example, Amazon EC2 [1] only sells pre-defined types of virtual machines (VMs). Despite their apparent simplicity, fixed-price policies inherently lack market agility and efficiency, failing to rapidly adapt to real-time changes in demand-supply relations. Consequently, both overpricing and underpricing routinely occur, which jeopardizes cloud providers' overall profits.

To effectively discover the market value of resources, auction mechanism design has been the focal point of recent literature on profit maximization, and suites of computationally efficient and truthful auction-style pricing mechanisms are proposed so that users can fairly compete for resources and the profit of cloud providers increases [9-13]. To simplify cloud providers' operations, periodically changed pricing policies are proposed in $[1,13]$. However, users may suffer as a result of this simplicity. For example, a user who submits a bid requesting a VM that lasts for more than one period faces the threat of being outbid and losing cloud usage in any one of the requested periods. As the price only changes periodically, fluctuations in supply-demand relations which are typically drastic due to the inherent dynamics and burst nature of user demands are not reflected in a timely and efficient manner.

More importantly, these auction mechanisms do not consider fault tolerance. Fault tolerance, an essential concept in cloud computing, refers to a service that is not interrupted when one or more servers fail. In the auction mechanism, once a bid is accepted, cloud providers must assign the VMs requested by the bids into servers, and each VM demands a certain amount of computing resources. VMs might not work if there is a server failure due to hardware or software problems. One efficient method to address the issue of failure is to create multiple replicas for the VMs in the system and to ensure that all replicas associated with one VM are dispersed to different servers. As a result, if one or more servers fail, the resources associated with the failed servers will be assigned to replicas hosted on the available servers. This process ensures that the total amount of resource assigned to the VM associated with the failed servers does not change even when one or more servers fail. Hence, this method can protect a system against server failure. More importantly, it requires each server to reserve a certain amount of each type of resource and ensures that assigning additional amount of 
resource to the replicas (as a result of server failure) will not result in overloaded servers.

In response to the limitations of the existing auction mechanisms, this paper proposes a novel and practical online auction mechanism for maximizing cloud providers' profits with considering the energy cost of servers. Firstly, in contrast to requiring users to specify a fixed amount of resource, our auction mechanism features an elastic model for time-varying user demands and allows users to request multiple types of resources. Furthermore, our auction mechanism ensures that at least one server in each assigned time slot for each accepted bid is available even when one or more servers fail.

However, the design of an online allocation algorithm for maximizing the profits of cloud providers in our setup is challenging. First, a decision on reserving resources for VMs should be made upon each bid, without knowledge of future information. Moreover, users are strategic and selfish; They want to gain unfair advantages via untruthfully revealing their preference for cloud resources. Cheating behaviors significantly degrade auction efficiency, and greatly discourage the participation of other users. Thus, it can be difficult to design a payment rule that works with the allocation algorithm to guarantee desired properties like truthfulness, which ensures that users always tell the real value of their bids to cloud providers. The classic Vickrey-Clarke-Groves (VCG) mechanism, essentially the only type of auction that guarantees both truthfulness and economic efficiency in offline settings [8], does not directly work in online settings because it requires computation of the exact optimal allocation to guarantee truthfulness. As this cannot be done in online settings without knowledge of future requests, the auction mechanism becomes more complex by considering fault tolerance and variation in user demands over time.

Our Contributions: In this paper, we design a truthful, fault tolerant, and time efficient online auctions for profit maximization with the expectation of good competitive ratios. First, we model the profit maximization problem using an Integral Linear Programming (ILP) optimization framework, which offers an elastic model for time-varying user demands. Second, we design an online auction mechanism consisting of a price-based allocation strategy and a pricing function. The online allocation strategy allocates multiple types of resource to each user while satisfying the time-varying user demands and ensuring at least one server is available for each user in each allocated time slot. In addition, the designed pricing function for updating marginal prices per unit resource plays a key role in achieving truthfulness and a good competitive ratio.

The rest of this paper is organized as follows. Sections II and III introduce the system model and problem definition, respectively. The online allocation strategy is described in Section IV. Section V proposes a pricing function, while the simulation results are shown in Section VI. We conclude the paper with Section VII.

\section{SYSTEM MODEL}

We adopt an online auction framework in an IaaS cloud. The cloud provider provides $R$ types of resources for users, such as CPU, RAM, and disk storage, and let $R$ represent $[1,2, \ldots, R]$. We assume that the maximum number of servers is $N$, which is fixed during time slots $[1,2, \cdots, T][1,14]$, and let $T$ represent $[1,2, \ldots, T]$. The maximum amount of type- $r, r \in R$ resource is $C^{r}$ at each time slot, while that of the $n^{t h}$ server is $c_{n}^{r}$. We assume that, in a cloud data center, power consumption increases linearly with the amount of utilized resources. The power cost consumed by unit type- $r, r \in R$ resource for one time slot is $\eta_{r}$ [6].

In addition, we assume that a user's bid arrives randomly in a large timespan. Note that multiple bids can arrive simultaneously and would be ordered randomly. Supposing that $I$ users participate in the auction, let $I$ represent set $[1,2, \ldots, I]$. Assume that user $i \in I$ arrives at time $t_{i}$ and requests a VM comprising a bundle of resources in a future usage duration and that the user's bid specifies: (i) $W_{i}$, the number of execution slots in which the VM works; and (ii) $d_{i}^{r}(j), j \in\left[1,2, \ldots, W_{i}\right]$, the amount of type- $r, r \in R$ resource to be occupied by the VM at the $j^{t h}$ execution slot. $d_{i}^{r}(j), r \in R, j \in$ $\left[1,2, \cdots, W_{i}\right]$ is specified in the bid based on its requirement at different times. Each bid also includes the start time $a_{i}$ to run the VM and the end time $d_{i}$, where $t_{i} \leq a_{i}<d_{i}$. Note that each bid execution does not need to be continuous; Moreover, user $i$ specifies a willingness-to-pay $b_{i}^{r}$ to receive one unit type- $r, r \in R$ resource for one time slot. Hence, the bid for user $i$ can be expressed as follows :

$$
B_{i}=\left\{W_{i}, a_{i}, d_{i}, d_{i}^{r}(j)_{r \in R, j \in\left[1, \ldots, W_{i}\right]}, b_{i}^{r}\right\}
$$

Let $b_{i}$ and $v_{i}$ represent the unit bid price and true unit valuation, where $b_{i}=\sum_{r=1}^{R} b_{i}^{r}$ and $v_{i}=\sum_{r=1}^{R} v_{i}^{r}$, and $v_{i}^{r}$ is the true unit type- $r, r \in R$ resource valuation for one time slot of bid $i$. The true unit valuation is the estimated payment by user $i$, and $v_{i}$ does not depend on other users' bids. We also assume that the true valuation $v_{i}$ is privately known to user $i$.

After bid $i$ appears, the cloud provider decides whether to accept bid $i$. If the bid is accepted, the provider allocates the execution slots for the requested VM and assigns the requested VM to servers. Let binary variable $x_{i}$ indicate whether bid $i$ is accepted. If the cloud provider accepts the $i^{t h}$ bid, $x_{i}$ equals 1 ; otherwise $x_{i}$ equals 0 . Let binary variable $y_{i}^{t}$ encode the scheduling of the $i^{t h}$ bid. If the cloud provider allows bid $i$ to be executed at time $t$, then $y_{i}^{t}$ equals 1 and the provider assigns at least $d_{i}^{r}(j)$ type- $r, r \in R$ resource for bid $i$, where $j=\sum_{t^{\prime}=1}^{t} y_{i}^{t^{\prime}}$; otherwise $y_{i}^{t}$ equals 0 and no resource is assigned to bid $i$. The cloud provider determines the payment $p(t, i)$ for user $i$ if the requested VM runs at time $t$, and the user $i$ is not charged for any slot in which the requested VM does not work. In addition, the cloud provider must ensure that the total number of allocated slots in which the VM requested by bid $i$ works is not less than $W_{i}$.

Importantly, to achieve the fault tolerance target, $f+1$ replicas of the VM requested bid $i$ are created when $y_{i}^{t}$ is equal to 1 , where $f$ is the maximum number of simultaneously failed servers. The amount of type- $r, r \in R$ resource assigned to each replica at time $t$ is $\frac{d_{i}^{r}(j) y_{i}^{t}}{f+1}, j=\sum_{t^{\prime}=1}^{t} y_{i}^{t^{\prime}}$. All replicas associated with one VM are placed on $f+1$ different servers. When a server fails, the replicas hosted on the available servers and associated with failed servers receive an additional amount of resource. Specifically, it is assumed that $m, 0 \leq m \leq f$ replicas of VM requested by bid $i$ at time $t$ are failed. Each replica associated with the VM requested by bid $i$ and hosted on an available server receives $\frac{m \times d_{i}^{r}(j)}{(f-m) \times(f+1)}, j=\sum_{t^{\prime}=1}^{t} y_{i}^{t^{\prime}}$ 

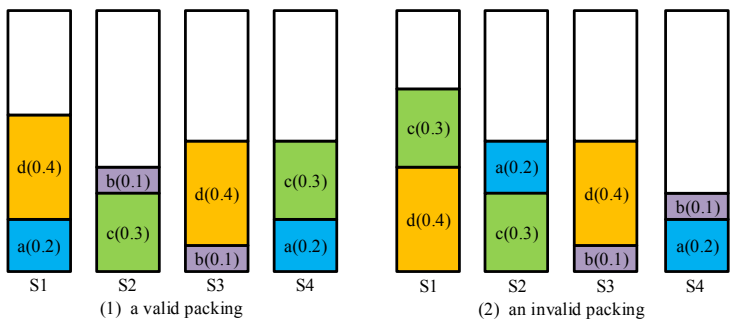

Figure 1: Valid and invalid assignments

type- $r, r \in R$ resource. This ensures that the total amount of type$r, r \in R$ resource assigned to bid $i$ does not change even when one or more servers fail.

More importantly, it must ensure that the assigning additional amount of resource to replicas (as a result of server failure) will not result in overloaded servers. For example, assume that all bidders only need storage service, and that the storage capacity of each server is 1 . Consider a bid sequence $\phi_{1}=<a=0.4, b=0.2, c=$ $0.6, d=0.8>$, and that each bid has two replicas. Two packing solutions for $\phi_{1}$ are shown in Fig.1. The solution on the left is a valid packing solution. The solution on the right is not valid since servers $S_{1}$ and $S_{3}$ share the storage service of bid $a$. If server $S_{3}$ fails, another 0.4 storage capacity will be assigned to the replica associated with bid $a$, which is hosted on $S_{1}$. Thus, the total capacity of server $S_{1}$ needs to be $0.4+0.3+0.4=1.1$, which is greater than the capacity of the servers.

All users are private and want to maximize their utility. If bid $i$ is accepted, the utility of user $i$, denoted by $u_{i}$, is the difference between the real valuation and payment; otherwise, $u_{i}$ equals 0 . Hence, utility $u_{i}$ can be calculated by equation (2) as follows:

$$
u_{i}= \begin{cases}\sum_{r=1}^{R} \sum_{j=1}^{W_{i}} v_{i}^{r} d_{i}^{r}(j)-\sum_{t \in T} p(t, i), & \text { if } x_{i}=1 \\ 0, & \text { if } x_{i}=0\end{cases}
$$

\section{PROBLEM FORMULATION}

In this paper we aim to solve the problem of maximizing the total profit of a cloud provider under a resource budget constraint, given the time-varying demands of users and considering the energy cost. At the same time, we try to achieve several important properties of auctions, such as truthfulness, fault tolerance, and computational efficiency.

Each type of resource of each server at each time slot can be divided into two separate parts: $\xi_{n}^{r}(t), \psi_{n}^{r}(t)$, where $\xi_{n}^{r}(t)$ is the amount of type- $r, r \in R$ resource occupied by replicas hosted on the $n^{t h}$ server at time $t$, and $\psi_{n}^{r}(t)$ is the reserved amount of type$r, r \in R$ resource for assigning additional amount of type- $r, r \in R$ resource to replicas hosted on the $n^{\text {th }}$ server and associated with failed servers at time $t$. Thus, the total amount of type-r, $r \in R$ resource that occupies the replicas at the $n^{t h}$ server at time $t$ is following equation (3).

$$
\xi_{n}^{r}(t)=\sum_{i \in \mathbb{B}_{t}} \frac{d_{i}^{r}(j) y_{i}^{t}}{f+1}, j=\sum_{t \prime=1}^{t} y_{i}^{t \prime}, t \prime \leq t .
$$

As mentioned above, to avoid service interruption in the execution slot, we create at least $f+1$ replicas for each VM at each assigned time slot and place them at different servers, such that no matter what $f$ server is faulty, one replica is still available. As a result, we have inequality (4). Inequality arises from the fact that there should always be enough reserved amount of type- $r, r \in R$ resource to accommodate the additional amount of type- $r$ resource to handle any $f$ failures at any time. Let us consider a particular scenario in which the first $f$ servers $\left(S_{1}\right.$ to $\left.S_{f}\right)$ are faulty at time $t$. In this case, we have $\sum_{n=1}^{f} \xi_{n}^{r}(t) \leq \sum_{n=f+1}^{N} \psi_{n}^{r}(t), r \in R$, where the left side is the total amount of type- $r$ resource requirement and should be no greater than the right side, which is the total reserved amount of type- $r$ resource of the remaining $N-f$ available servers. As any $f$ servers can fail, we have $\left(\begin{array}{l}N \\ f\end{array}\right)$ similar inequalities for each resource at any time. Inequality (4) is derived by summing all of these inequalities.

$$
\sum_{n=1}^{N} \xi_{n}^{r}(t)\left(\begin{array}{c}
N-1 \\
f-1
\end{array}\right) \leq \sum_{n=1}^{N} \psi_{n}^{r}(t)\left(\begin{array}{c}
N-1 \\
f
\end{array}\right), r \in R, t \in T .
$$

Hence, the total amount of type- $r, r \in R$ resource occupied by replicas at time $t$, denoted by $\Xi_{r}^{t}$, is $\sum_{n=1}^{N} \psi_{n}^{r}(t)$. The total amount of type- $r, r \in R$ resource reserved for replicas at time $t$ is represented by $\Psi_{r}^{t}$, which is equal to $\sum_{n=1}^{N} \xi_{n}^{r}(t)$. Thus, the total active amount of type- $r, r \in R$ resource at time $t$ is denoted by $\Omega_{r}^{t}$, which is equal to $\Xi_{r}^{t}+\Psi_{r}^{t}$.

The profit of the cloud provider is the total received payment of selling time $t^{\prime} s$ resources minus the power cost of time $t$, integrated over the time window $[1, T]$. With this observation, we define the profit maximization problem as follows:

$$
\max \left(\sum_{t=1}^{T}\left[\sum_{i=1}^{I} p(t, i)-\sum_{r}^{R} \eta_{r} \Omega^{r}(t)\right]\right)
$$

s.t.

$$
\begin{gathered}
y_{i}^{t} \leq x_{i}, \forall i \in I, \forall t \in T \\
\sum_{t=a_{i}}^{d_{i}} y_{i}^{t} \geq W_{i}, \forall i \in I, \forall t \in T \\
\sum_{n=1}^{N} \beta_{i}^{n}(t) \geq(f+1) \times y_{i}^{t}, \forall i \in I, \forall t \in T \\
\xi_{n}^{r}(t)=\sum_{i \in I}\left(d_{i}^{r}\left(j_{i}\right) y_{i}^{t}\right) /(f+1), \forall i \in I, \forall t \in T, \forall r \in R \\
\sum_{n=1}^{N} \xi_{n}^{r}(t)\left(\begin{array}{l}
N-1 \\
f-1
\end{array}\right) \leq \sum_{n=1}^{N} \psi_{n}^{r}(t)\left(\begin{array}{c}
N-1 \\
f
\end{array}\right), \forall t \in T, \forall r \in R \\
\xi_{n}^{r}(t)+\psi_{n}^{r}(t) \leq c_{n}^{r}, \forall t \in T, \forall r \in R, \forall n \in N \\
\sum_{r=1}^{R} b_{i}^{r} d_{i}^{r}\left(j_{i}\right)-p(t, i) \geq 0, \forall i \in I, \forall t \in T
\end{gathered}
$$




$$
\begin{gathered}
j_{i}=\sum_{t \prime=1}^{t} y_{i}^{t \prime}, \forall i \in I, \forall t \in T \\
y_{i}^{n}(t, j) \leq x_{i} \in\{0,1\}, \forall i \in I, \forall t \in T
\end{gathered}
$$

Objective (5) maximize the total profit with considering the energy cost. Constraints $(5 a)$ and $(5 b)$ guarantee that if bid $i$ is accepted, then the cloud provider must assign sufficient time slots between the arrival time $a_{i}$ and deadline $d_{i}$ to serve it; otherwise, no execution slot will be assigned for bid $i$. Let binary variable $\beta_{i}^{n}(t)$ assign the replicas of the VM requested by bid $i$ to the server $n$ at time $t$. If server $n$ hosts one replica of the VM requested by bid $i$ at time $t$, $\beta_{i}^{n}(t)$ is equal to 1 ; otherwise, $\beta_{i}^{n}(t)$ is equal to 0 . Moreover, if the VM requested by bid $i$ runs at time $t$, constraint $(5 c)$ ensures that at least $f+1$ replicas are created and that each replica is placed at a different server. The amount of type- $r, r \in R$ resource assigned to each replica at time $t$ is $\frac{d_{i}^{r}(j) y_{i}^{t}}{f+1}, j=\sum_{t^{\prime}=1}^{t} y_{i}^{t^{\prime}}$, which is ensured by constraint $(5 d)$. Constraint ( $5 e$ ) ensures that there is always enough reserved amount of type- $r$ resource to handle any $f$ failures at any time. The capacity of the resource is formulated in constraint $(5 f)$. Constraint (5g) ensures that the bidding price is greater than the required payment.

Even in an offline setting, without constraints $(5 c-5 e)$ and without considering fault tolerance, ILP (5) is still a NP-hard combination optimization problem, equivalent to the classic knapsack problem. The challenge further escalates when we involve fault tolerance and pursue online decision-making with consideration of variation in the amount of requested resource with time.

\section{ONLINE AUCTIONS FOR PROFIT MAXIMIZATION WITH A RESOURCE ALLOCATION STRATEGY}

In this section, to maximize the profit of the cloud provider, we propose an Online Dynamic Resource Allocation Strategy (ODRAS) with consideration of $(5 a-5 i)$. One feature of this online algorithm is that it charges the lowest payment for each user while satisfying the time-varying resource demands and fault tolerance requirement. Let $p_{i}(t, w)$ represent the payment of user $i$ at the $t^{t h}$ time slot assuming assignment of the $w^{t h}$ execution slot of user $i$ to the $t^{t h}$ time slot, and $p_{i}(t, w)$ follows $\sum_{r=1}^{R} \sum_{\Xi_{r}(t,-i)}^{\Xi_{r}(t,-i)+d_{i}^{r}(w)} \phi_{r}(l)$, where $\Xi_{r}(t,-i)$ represents the total amount of type- $r$ resource allocated to the replicas at time $t$ before bid $i$ appears. $\phi_{r}(l)$ represents the marginal price of the type- $r$ resource and $\phi_{r}(l)$ is bid-independent and nondecreasing. In addition, let $S_{i}(w, t), 0<w \leq W_{i}, a_{i} \leq t \leq d_{i}$ denote the total payment of $w$ continuous slots of bid $i$ with the last slot being at the $t^{t h}$ time slot. Note that $S_{i}(0, t), t \in\left[a_{i}, d_{i}\right]$ is equal to 0 . Thus $S_{i}(w, t), a_{i} \leq t \leq d_{i}$ can then be represented as equation (6).

$$
S_{i}(w, t)= \begin{cases}S_{i}(w-1, t-1)+p_{i}(t, w), & 0<w \leq W_{i} \\ 0, & w=0\end{cases}
$$

where $p_{i}(t, w)=\sum_{r=1}^{R} \sum_{\Xi_{r}(t,-i)}^{\Xi_{r}(t,-i)+d_{i}^{r}(w)} \phi_{r}(l)$.

If the $w^{t h}$ execution slot of user $i$ is assigned to the $t^{t h}$ time slot, fault tolerance must be considered. To achieve this target, we propose Fault Tolerance (FT) algorithm and the details of FT are as follows: Firstly, we introduce two sets, $X_{n}^{t}$ and $L_{i}^{t}$. $X_{n}^{t}$ includes the labels of bids in which one replica of the bid is packed in the $n^{t h}$ server at time $t . L_{i}^{t}$ represents the labels of the servers that pack the replicas of bid $i$ at time $t$. In addition, let $\alpha_{i, w}^{t}$ denote the label set of the servers placing the replicas of the VM requested by bid $i$, assuming that the $w^{t h}$ execution slot of user $i$ is assigned to the $t^{t h}$ time slot. Initially, $\boldsymbol{\alpha}_{i, w}^{t}$ is an empty set.

FT first creates $f+1$ replicas for the VM requested by bid $i$ and each replica is assigned $\frac{d_{i}^{r}(w)}{f+1}$ type- $r, r \in R$ resource. FT also finds servers in which the remaining amount of each type of resource is larger than the demand of user $i$ at time $t$ and puts the labels of the servers satisfying above constraint into set $\Phi_{i}^{t}$. Initially, $\Phi_{i}^{t}$ is an empty set. If the $n^{t h}$ server belongs to $\Phi_{i}^{t}$ at time $t$, then it must satisfy the constraint that the remaining amount of type- $r, r \in R$ resource is no less than 0 , assuming that one replica of the VM is packed into it. Mathematically, if the $n^{t h}$ server is in $\Phi_{i}^{t}$, it must satisfy formulations (7) and (8).

$$
\begin{gathered}
c_{n}^{r}-\left(\xi_{n}^{r}(t)+\frac{d_{i}^{r}(w)}{f+1}+\Delta_{n}^{t}\right) \geq 0, \forall r \in R \\
\Delta_{n}^{t}=\max \left(\psi_{n}^{r}(t), f * \frac{d_{i}^{r}(w)}{f+1}\right), \forall r \in R
\end{gathered}
$$

When placing one replica of the VM into a server with label $\phi, \phi \in \Phi_{i}^{t}$, the energy consumption of the server increases. In addition, $\xi_{\phi}^{r}(t)$ and $\psi_{\phi}^{r}(t), r \in R$ are updated. $\xi_{\phi}^{r}(t)$ will increase to $\xi_{\phi}^{r}(t)+d_{i}^{r}(w) /(f+1), r \in R$. Since all replicas associated with one VM must appear at different servers such that $\psi_{\phi}^{r}(t)$ is at least the maximum between $\psi_{\phi}^{r}(t), r \in R$ and the reserved amount of type- $r$ resource for bid $i$ needed at time $t$, which is equal to $f * \frac{d_{i}^{r}(w)}{f+1}, r \in R$. Mathematically, $\xi_{\phi}^{r}(t)$ and $\psi_{\phi}^{r}(t)$ are updated based on equations (9) and (10), respectively.

$$
\begin{gathered}
\xi_{\phi}^{r}(t)=\xi_{\phi}^{r}(t)+\frac{d_{i}^{r}(w)}{f+1}, \forall r \in \boldsymbol{R} \\
\psi_{\phi}^{r}(t)=\max \left(\psi_{\phi}^{r}(t), f \times \frac{d_{i}^{r}(w)}{f+1}\right), \forall r \in \boldsymbol{R}
\end{gathered}
$$

As mentioned in Section II, power consumption increases linearly with the amount of utilized resources [6]. Thus, the increased energy consumption of the $\phi^{t h}$ server at the $t^{t h}$ time slot is denoted by $e_{\phi}^{t}$, and follows equation (11).

$$
e_{\phi}^{t}=\sum_{r=1}^{R} \eta_{r}\left(\frac{d_{i}^{r}(w)}{f+1}+\max \left(\psi_{\phi}^{r}(t), f \times \frac{d_{i}^{r}(w)}{f+1}\right)-\psi_{\phi}^{r}(t)\right)
$$

FT will choose the server from $\Phi_{i}^{t}$ that results in the smallest increase in energy consumption. Let $s$ denote the label of the selected server. FT then places $s$ into $\boldsymbol{\alpha}_{i, w}^{t}$.

Next, FT deletes the servers that share the same bid with server $s$ from $\Phi_{i}^{t}$. As a specific example, consider two sets, $\boldsymbol{A}$ and $\boldsymbol{B}$, and let $\boldsymbol{A}-\boldsymbol{B}$ represent the elements belonging to $\boldsymbol{A}$ but not $\boldsymbol{B}$. The updated available servers is set to $\Phi_{i}^{t}-L_{j}^{t}, j \in X_{s}^{t}$. Note that the available servers list $\Phi_{i}$ might be an empty set after updating. This means that assigning the $w^{\text {th }}$ execution time slot of bid $i$ into time slot $t$ does not satisfy the fault tolerance constraint. Next, FT makes $p_{i}(w, t)$ 
equal to $+\infty$ and sets $\boldsymbol{\alpha}_{i, w}^{t}$ as an empty set. The above process is then repeated until all replicas associated with the VM are packed or $p_{i}(w, t)$ is equal to $+\infty$. Importantly, there may initially be no available servers for the VM; in this case, FT also makes $p_{i}(w, t)$ equal to $+\infty$ and sets $\boldsymbol{\alpha}_{i, w}^{t}$ as an empty set. Therefore, $p_{i}(w, t)$ can be rewritten as equation (12).

$$
p_{i}(t, w)=\left\{\begin{array}{lr}
\sum_{r=1}^{R} \sum_{\Xi_{r}(t,-i)}^{\Xi_{r}(t,-i)+d_{i}^{r}(w)} \phi_{r}(l), \text { if } \boldsymbol{\alpha}_{i, w}^{t} \neq \emptyset \\
+\infty, & \text { if } \boldsymbol{\alpha}_{i, w}^{t}=\emptyset
\end{array}\right.
$$

The pseudocode of achieving the fault tolerance target is shown in Algorithm2.

Let $P_{i}(w, t), 0 \leq w \leq W_{i}$ be the lowest payment for bid $i$ with its first $w$ execution slots allocated before the $t^{t h}$ time slot, and set $D_{i}(w, t), 0 \leq w \leq W_{i}$ as the lowest payment for bid $i$ with the first $w-1$ execution slots allocated before the $(t-1)^{t h}$ time slot and the last execution slot assigned at the $t^{t h}$ slot. From this definition, we can derive that $D_{i}(w, t)$ is the minimization of the sum of $P_{i}(w-j, t-1)$ and $S_{i}(j, t)$ with $j$ varying from 0 to $w$. Mathematically, $D_{i}(w, t)$ can be calculated by equation (13).

$$
D_{i}(w, t)=\min _{j=0 \ldots w}\left(P(w-j, t)+S_{i}(j, t)\right) .
$$

Meanwhile, $P_{i}(w, t)$ is the minimization value of $D_{i}\left(w, t^{\prime}\right)$ with $t^{\prime}$ varying from $a_{i}$ to $t$, which is represented by equation (14).

$$
P_{i}(w, t)=\min _{t,=a_{i} \ldots t}\left(D_{i}(w, t \prime)\right) .
$$

Next, we can get the minimization payment $P_{i}\left(W_{i}, d_{i}\right)$ by recursively resolving $P_{i}(w, t), 0 \leq w \leq W_{i}$ and $D_{i}(w, t), 0 \leq w \leq W_{i}$. If $P_{i}\left(W_{i}, d_{i}\right)$ is less than $\sum_{r=1}^{R} \sum_{j=1}^{W_{i}} d_{i}^{r}(j) b_{i}^{r}$, then ODRAS accepts bid $i$; otherwise, bid $i$ is rejected. Accordingly, the binary variables $y_{i}^{t}$ and $\beta_{i}^{n}(t)$ are determined. The pseudocode of ODRAS and FT are shown in Algorithm 1, 2 respectively.

Lemma 1: No two servers share replicas of more than one VM, which is placed by the FT algorithm at each time slot.

Proof:

Consider any bid $i$. If bid $i$ is assigned at time $t$, ODRAS must select a server for packing the replicas of bid $i$ only in $\Phi_{j}^{t}$. After a replica is packed into server $s$, ODRAS deletes the servers sharing the same bid with server $s$ from $\Phi_{j}^{t}$. Therefore no two servers share replicas of more than one VM in the ODRAS algorithm at each time slot.

We then validate the FT algorithm using Lemma 1.

Theorem 1. In the schemes resulting from FT, no server is overloaded in the case of failure of at most $f$ servers at each time slot.

Proof:

Consider an arbitrary server $S_{n}^{t}$ in the FT at time $t$ and a server set $S_{-n}^{t}$, where $S_{-n}^{t}$ consists of any $f$ servers but does not include server $S_{n}^{t}$. Let $\lambda_{n}^{t}$ be the replica packed in $S_{n}^{t}$ which occupies the most type- $r, r \in R$ resources among all replicas packed in $S^{n}$ at time $t .\left|\lambda_{n}^{t}\right|^{r}$ represents the amount of type- $r, r \in R$ resource assigned to $\lambda_{n}^{t}$. We show that, in case of simultaneous failure of all servers in $S_{-n}^{t}$, the extra resource assigned to the replicas hosted on $S^{n}$ does not cause overload. By Lemma 1 , server $S_{n}^{t}$ and the servers in $S_{-n}^{t}$ share at most one replica. Thus, the additional type- $r, r \in R$ resource assigned to $S^{n}$ is at most $f \times\left|\lambda_{n}^{t}\right|^{r}$. As previously mentioned, once the replica is packed into a server, the reserved space of the server is updated following (10). This implies that the reserved type- $r, r \in \boldsymbol{R}$ resource in server $S_{n}^{t}$ is at least $f \times\left|\lambda_{n}^{t}\right|^{r}$. Therefore, there is no overflow for $S^{n}$ when all servers in $S_{-n}^{t}$ fail simultaneously. $\square$

\section{DEFINING THE MARGINAL PRICE FUNCTION AND DERIVING THE COMPETITIVE RATIO}

In the following section, we present the marginal price function and the competitive ratio with respect to the profit of the cloud provider.

Theorem 2 : It is assumed that, among the unit bid prices of all submitted bids, the unit bid price is at most $\alpha$ times the lowest unit bid price. ODRAS has a competitive ratio of $\alpha \cdot \gamma$, when the marginal price function of each type-r resource is defined as follows: $\phi_{r}(l)=\eta_{r}+\eta_{r} \cdot\left(\gamma_{r}-1\right) \cdot e^{l \gamma_{r} / C^{r}} \cdot \gamma_{r}$ is the solution to equation $\phi_{r}\left(C^{r}\right)=C^{r} b_{\text {max }} . b_{\max }$ is the highest unit bid price and $C^{r} b_{\max }$ is the highest price that users are willing to pay for a type- $r$ resource. $\gamma$ is equal to $\max _{r \in R} \gamma_{r}$.

\section{Proof:}

To deduce the competitive ratio, we should compare the profit under our online mechanism with the offline optimal profit. This is because the payment of each user is less than the valuation of their bid. The offline optimal social welfare, which is sum of the provider's profit and the user' aggregate utility, and social welfare is the upper bound of the offline optimal profit. The social welfare under the offline VCG mechanism is the optimal social welfare. Therefore, we compare the profit under our online mechanism with the social welfare under the offline VCG mechanism [7].

Recall that, under our mechanism, the total type- $r$ resource occupied by the replicas at time $t$ is $\Xi_{r}^{t}$, and that reserved for the replicas at time $t$ is $\Psi_{r}^{t}$. $\Omega_{r}^{t}$ denotes the total active amount of type$r$ resource at time $t$, and $\Omega_{r}^{t}=\Xi_{r}^{t}+\Psi_{r}^{t}, r \in R, t \in T$. Thus, the profit of the cloud provider at time $t$ is following equation (15).

$$
P(t)=\sum_{r=1}^{R}\left(\sum_{l=1}^{\Xi_{r}^{t}} \phi_{n}(l)-\eta_{r} \Omega_{r}^{t}\right)
$$

We next discuss the offline VCG mechanism. Let $\mathbb{B}_{v c g}$ represent the accepted bids, where bid $i \in \mathbb{B}_{v c g}$ is assigned $d_{i}^{r}$ type- $r, r \in R$ resource at time $t$ under the offline VCG mechanism. The social welfare and payment obtained from the offline VCG mechanism at time $t$ are denoted as $P_{v c g}(t)$ and $W_{v c g}(t)$, respectively. The offline VCG mechanism is based on the complete information of all bids. Under the VCG mechanism, the unit valuation of each bid is equal to unit bidding price. As a result, the social welfare under the offline VCG mechanism equals the aggregate bidding price of the winning bids minus the server costs. Thus, the social welfare for selling resources at time $t$ under the offline VCG mechanism is as follows:

$$
W_{v c g}(t)=\sum_{i \in \mathbb{B}_{v c g}} \sum_{r=1}^{R} \sum_{j=1}^{W_{i}} d_{i}^{r}(j) b_{i}^{r}-\sum_{r=1}^{R} \eta_{r} \Omega_{r}^{t, v c g}
$$

where $\Omega_{r}^{t, v c g}$ denotes the total active type- $r$ resource at time $t$ in the offline VCG mechanism. The unit price of each winning bid is smaller than or equal to the claimed unit bid price. Among the bid 
prices of all accepted bids, if the highest unit bid price is at most $\alpha$ times the lowest unit bid price, the social welfare $W_{v c g}(t)$ is smaller than or equal to $\alpha$ times the total payment $P_{v c g}(t)$.

Let $b_{\mathrm{min}}^{v c g}$ denote the smallest unit bid price among the unit bid prices of all accepted bids. Thus, we get inequality $b_{\min }^{v c g} \leq$ $\sum_{r=1}^{R} \phi_{r}\left(\Xi_{r}^{t}\right)$. This inequality arises because the upper bound of the social welfare under the offline VCG mechanism is $W_{v c g}^{u}$, where $W_{v c g}^{u}$ is following equation (17)

$$
\sum_{i \in \mathbb{B}_{v c g}} \sum_{r=1}^{R} \sum_{j=1}^{W_{i}} b_{i}^{r} \cdot d_{i}^{r}(j) .
$$

In addition, the winning bids in ODRAS are all satisfied in the offline VCG auction mechanism. If $b_{\min }^{v c g} \geq \sum_{r=1}^{R} \phi_{r}\left(\Xi_{r}^{t}\right)$, this contradicts the fact that the social welfare under the offline VCG mechanism is larger than the profit. As a result, we get inequality (18).

$$
P_{v c g}(t) \leq v_{x}^{v c g} \sum_{r=1}^{R} C^{r} \leq \sum_{r=1}^{R} \phi_{r}\left(\Xi_{r}^{t}\right) C^{r}
$$

We denote the offline optimal profit as $P_{\text {opt }}(t)$. Because $P_{\text {opt }}(t) \leq$ $W_{v c g}(t) \leq \alpha P_{v c g}(t)$, we can obtain $\frac{P_{o p t}(t)}{P(t)} \leq \frac{\alpha P_{v c g}(t)}{P(t)}$.

$$
\begin{gathered}
\frac{P_{o p t}(t)}{P(t)} \leq \frac{\alpha \sum_{r=1}^{R} \phi_{r}\left(\Xi_{r}^{t}\right) C^{r}}{\sum_{r=1}^{R}\left(\sum_{l=1}^{\Xi_{r}^{t}} \phi_{r}(l)-\eta_{r} \Xi_{r}^{t}\right)} \\
\frac{P_{o p t}(t)}{P(t)} \leq \frac{\alpha \phi_{r}\left(\Xi_{r}^{t}\right) C^{r}}{\sum_{r=1}^{\Xi_{r}^{t}} \phi_{r}(l)-\eta_{i} \Xi_{r}^{t}}
\end{gathered}
$$

In formula (18), we define $r=\arg \max \frac{\phi_{r}\left(\Xi_{r}^{t}\right)}{\sum_{l=1}^{\Xi_{r}^{t}} \phi_{r}(r)-\eta_{i} \Xi_{r}^{t}}$. Thus, we have inequality (21).

$$
\frac{\phi_{r}\left(\Xi_{r}^{t}\right) C^{r}}{\sum_{l=1}^{\Xi_{r}^{t}} \phi_{r}(l)-\eta_{r} \Xi_{r}^{t}} \leq \frac{\phi_{r}\left(\Xi_{r}^{t}\right) C^{r}}{\int_{0}^{\Xi_{r}^{t}} \phi_{r}(l) d l-\eta_{r} \Xi_{r}^{t}}
$$

Let the right part of the above inequality equal $\gamma_{r}$ so that we get the marginal price function as follows :

$$
\phi_{r}(l)=\eta_{r}+\eta_{r} \cdot\left(\gamma_{r}-1\right) \cdot e^{l \gamma_{r} / C^{r}}
$$

In the above equality, $\gamma_{r}$ is the solution to the equation $\phi_{r}\left(C^{r}\right)=$ $C^{r} b_{\text {max }}$. Let $\gamma=\max _{r \in R} \gamma_{r}$. Thus, we get the upper bound of the competitive ratio as $\alpha \cdot \gamma$.

\section{THEORETICAL ANALYSIS}

This section presents the theoretical analysis that shows that our online auction mechanism is truthful and computationally efficient.

Theorem 3: ODRAS is truthful.

Proof: The truthfulness of a cloud auction refers to the fact that the dominant strategy for each user is to report the true valuation and true information (e.g., bid arrival time) in their bid, which always maximizes utility regardless of other users' bids.

In our auction mechanism, the marginal prices are independent of the bidding price for any option in each bid. Further, we always assign bids to servers to maximize each bid's utility given the current marginal price. Upon the arrival of each bidder, we always charge users the lowest payment that yields the maximal utility for the bidder and accept the bid if the utility is positive. In this way, our mechanism is equivalent to a sequential posted price mechanism [4] when an auctioneer posts the prices and lets the bidders maximize utility. Under this take-it-or-leave-it pricing scheme, a bidder cannot improve utility by lying about the price for their bid [4]. If the bidding price is higher than the corresponding true value, the bidder may suffer from negative utility; if the bidding price is lower than the true value, this bid may not be selected, resulting in lower utility for the bidder. Therefore, even for a multi-minded bidder, reporting truthful bidding prices is the dominant strategy.

(Truthfulness in arrival time): The marginal prices are nondecreasing in the amount of allocated resources, which is nondecreasing over the resource allocation time. Hence, a bidder cannot decrease the total price of the resource that it requests by delaying its arrival. Note that the arrival time of a bid is the first time that the bidder is aware of their demands, and so the arrival time cannot be earlier.

(Truthfulness in resource occupation times): Dropping part of the true resource occupation duration in the request risks failure to complete the job. However, the marginal prices are non-negative according to (22). Thus, requesting a superset of the true resource occupation duration increases a bidder's payment and decreases their utility. $\square$

Theorem 4: The online auction mechanism has a polynomial time complexity of $O(T \times T \times((f+1) \times T)+2 T)$.

Proof: As our mechanism is online, we only need to analyze the computational complexity for the user. The for loop in Algorithm 2 continues at most $f+1$ times. The first and last for loop in Algorithm 1 continue at most $T$ times, and the iteration represented by lines 11-21 in Algorithm 1 runs at most $T \times T \times((f+1) \times T)$ times. Therefore, the time complexity of our online auction mechanism is $O(T \times T \times((f+1) \times T)+2 T) . \square$

\section{SIMULATION}

We evaluate our method through trace-driven simulation studies. We exploit the trace of Google Cluster Data , which contains information for each job such as execution duration and resource demands (CPU and RAM). We translate each job into a bid. The total number of users is 600 . The unit bid price is randomly picked within the range $\left[v_{\min }, v_{\max }\right]$, in which $v_{\min }$ is $5.5 \$$ and $v_{\max }$ varies in different experiments. The power costs for one CPU and $1 \mathrm{~GB}$ memory in each time slot are $0.016 \$$ and $0.0028 \$$, respectively. Each experiment is run 20 times and we use the average of all runs as the final result.

We compare our online auction mechanism with two online auction mechanisms called Greedy and Lazy [2], respectively. They both consist of an allocation rule and a payment rule. The execution slots for bid $i$ are close to $a_{i}$ in Greed algorithm. While the execution slots allocated by Lazy are close to $d_{i}$. In addition, both Greedy and Lazy apply equation (22) to their price function and adopt Mirror [5] algorithm to achieve fault tolerance target.

The profits achieved by ODRAS, Greedy, and Lazy are shown in in Fig. 2, Fig. 3, and Fig. 4, respectively. These three figures plot the total profits under different numbers of requesting bids and different values of $\alpha$. From Fig.2, Fig. 3 and Fig4, we can see that the total profit of the ODRAS strategy is higher than that of both Greedy and Lazy, and that the number of requesting bids and value 


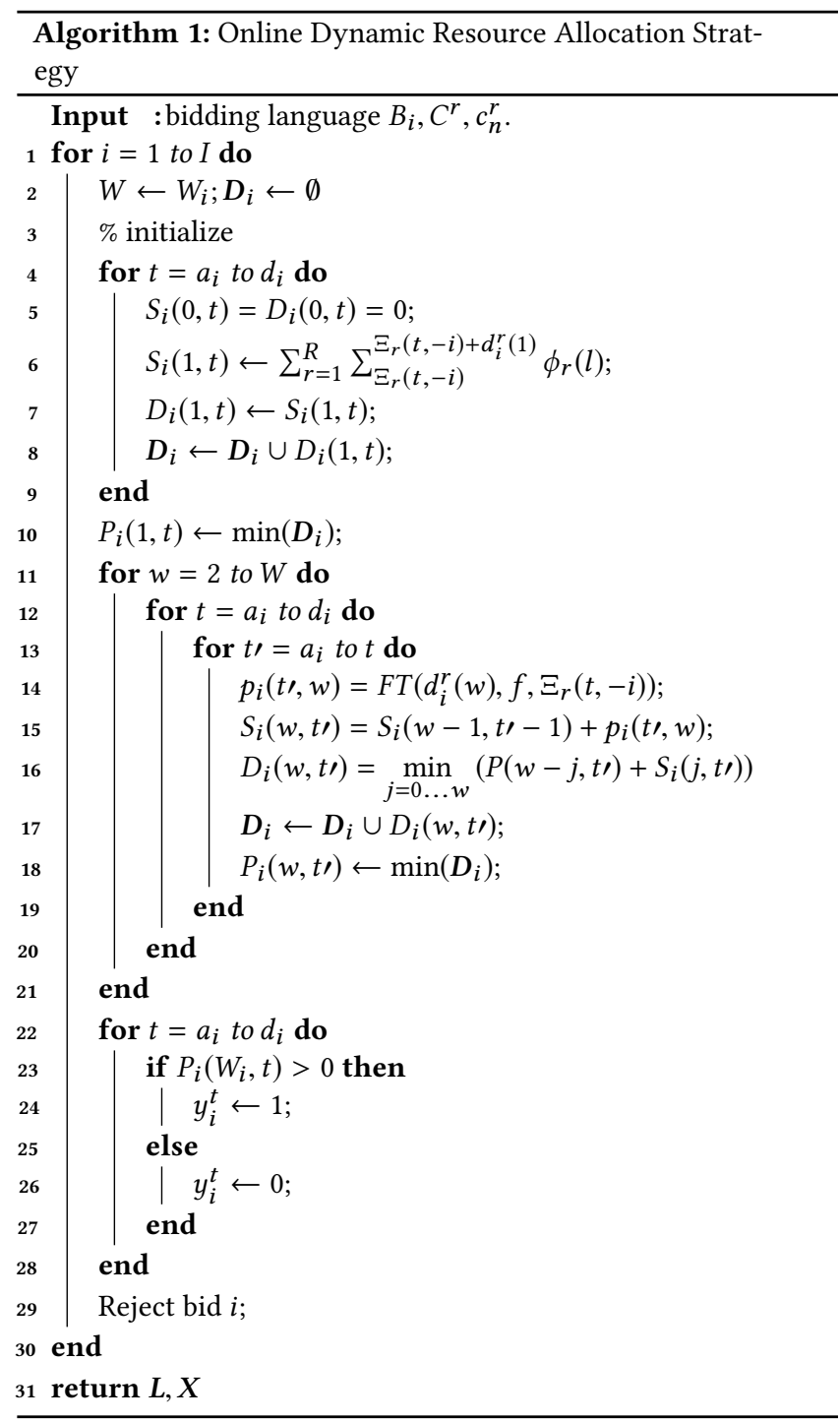

of $\alpha$ influence the profit. The reason for this is as follows: From Fig.5, Fig.6 and Fig7, we can see that the number of accepted bids increases as the number of requesting bids increases, and that the number of bids accepted by ODRAS is higher than that for Greedy and Lazy. As a result, the profit of ODRAS is higher than that of Greedy and Lazy. In addition, as the bidding price rises with an increase of $\alpha$, the high value bids lead to higher profit. However, from Figs. 5-7, we can see that the numbers of accepted bids are similar for different $\alpha$ values.

The number of occupied servers is shown in Fig.8, Fig.9 and Fig10. From Figs. 5-7, we can see that the number of bids accepted by ODRAS is higher than that accepted by Greedy and Lazy. However, the number of servers opened by ODRAS is less than that opened by Greedy and Lazy. This is because Greedy and Lazy adopt the extended Mirror algorithm to achieve fault tolerance. The extended Mirror algorithm requires each server to reserve $\frac{f}{f+1}$ capacity; thus, even when $f$ is $1,50 \%$ of the capacity is reserved for receiving the

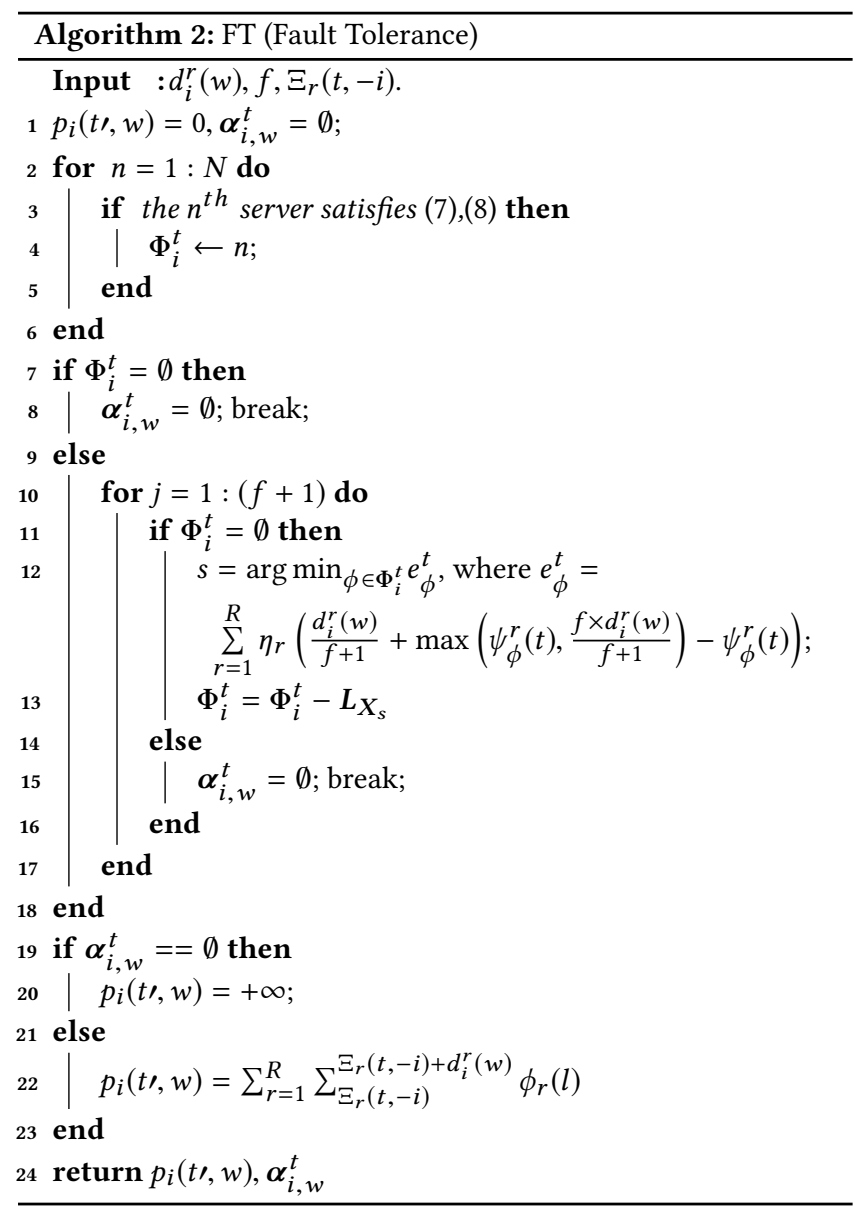

failed workload. As $f$ increases, the extended Mirror algorithm requires more capacity to achieve the fault tolerance target. In ODRAS, the reserved space for each server is only $f$ times the maximum size of the replica packing in the server. In the worst case scenario where all replicas have the same size, $\frac{1}{f+1}$, ODRAS needs a reserve of $\frac{f}{f+1}$; otherwise, the reserved space in each server is less than $\frac{f}{f+1}$. As a result, ODRAS can accept more bids with less servers relative to Greedy and Lazy.

\section{CONCLUSION}

This paper examined the important problem of designing an online auction mechanism. To address the limitations of the existing auction mechanisms for provisioning VMs in IaaS clouds and achieving the fault tolerance target, we designed a truthful online auction mechanism with the following features: 1) an elastic model for inputting time-varying user demands and allowing users to request multiple types of resources; and 2) assurance that at least one server for each accepted bid is available even when one or more servers fail. Our online auction mechanism has two main building blocks, namely a price-based allocation rule and a payment rule. The allocation rule allocates a time-varying VM fleet to each user while 


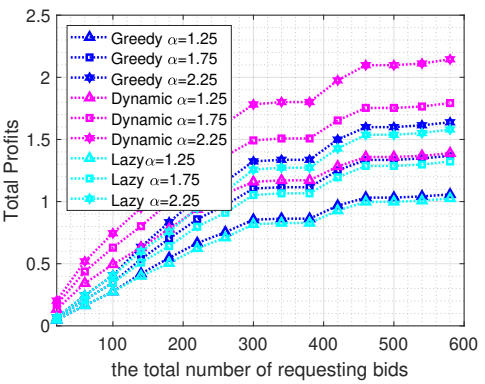

Figure 2: total profit with $f=1$

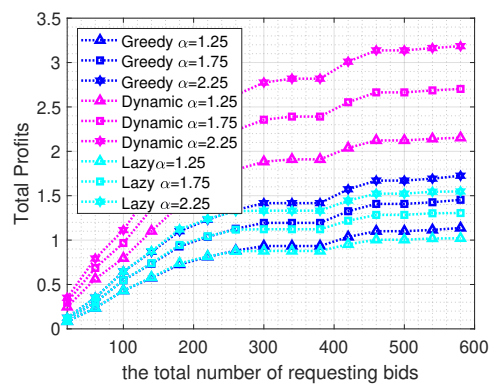

Figure 3: total profit with $f=2$

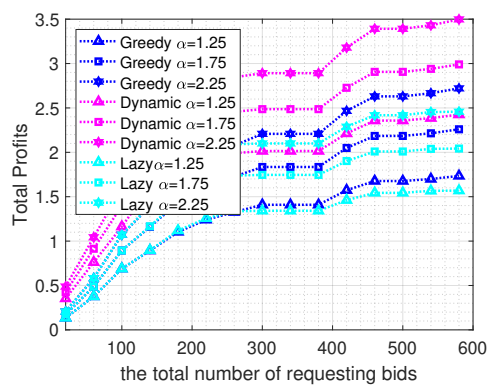

Figure 4: total profit with $f=3$
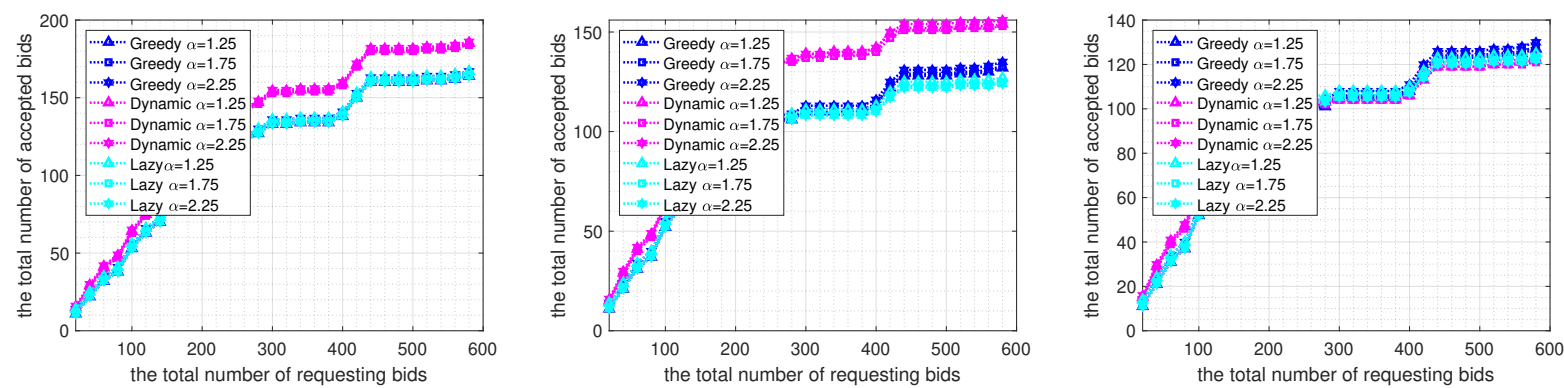

Figure 5: total number of accepted Figure 6: total number of accepted Figure 7: total number of accepted bids with $f=1$ bids with $f=2$ bids with $f=3$
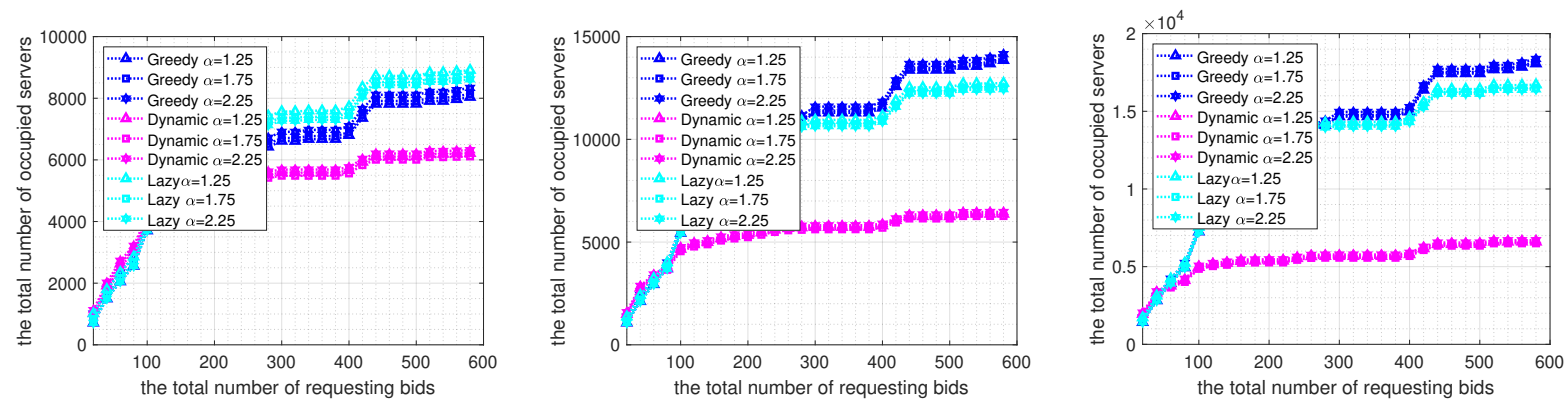

Figure 8: total number of opened Figure 9: total number of opened Figure 10: total number of opened servers with $f=1$ servers with $f=2$ servers with $f=3$

satisfying the specified elastic demand and fault tolerance requirement. The payment rule determines the payment that each user pays the cloud provider. We designed a marginal price function for each VM type, which is proved to achieve a competitive ratio. We also carried out solid theoretical analysis and trace-driven simulations to show that our online auction mechanism achieves truthfulness, individual rationality, and high computational efficiency.

\section{ACKNOWLEDGMENT}

We would like to acknowledge the financial support provided by the China Scholarship Council (NO.201806250168) during Boyu Li's visit to Nanyang Technological University, and this work is partially sponsored by National Key R\&D Program of China (No.
2019YFB2101700, 2018YFB0804402), National Science Foundation of China (61902276), the Key Research and Development Project of Sichuan Province (No.21SYSX0082).

\section{REFERENCES}

[1] February 4, 2021. Amazon Elastic Compute Cloud. https://aws.amazon.com/ec2/ ?nc1=h_ls.

[2] February 4, 2021. tech report. https://drive.google.com/file/d/ 1Q4jh6XcgkNS5GOBGWQM7lsn-19yiHfCZ/view?usp=sharing

[3] Sivadon Chaisiri, Bu-Sung Lee, and Dusit Niyato. 2012. Optimization of resource provisioning cost in cloud computing. IEEE Transactions on Services Computing 5, 2 (2012), 164-177.

[4] Shuchi Chawla, Jason D Hartline, David L Malec, and Balasubramanian Sivan. 2010. Multi-parameter mechanism design and sequential posted pricing. In Proceedings of the forty-second ACM symposium on Theory of computing. 311-320. 
[5] Khuzaima Daudjee, Shahin Kamali, and Alejandro López-Ortiz. 2014. On the online fault-tolerant server consolidation problem. In Proceedings of the 26th ACM symposium on Parallelism in algorithms and architectures. 12-21.

[6] Bhavani Krishnan, Hrishikesh Amur, Ada Gavrilovska, and Karsten Schwan. 2011. VM power metering: feasibility and challenges. ACM SIGMETRICS Performance Evaluation Review 38, 3 (2011), 56-60.

[7] Juan Li, Yanmin Zhu, Jiadi Yu, Chengnian Long, Guangtao Xue, and Shiyou Qian. 2018. Online auction for IaaS clouds: Towards elastic user demands and weighted heterogeneous VMs. IEEE Transactions on Parallel and Distributed Systems 29, 9 (2018), 2075-2089.

[8] Ahuva Mu'Alem and Noam Nisan. 2008. Truthful approximation mechanisms for restricted combinatorial auctions. Games and Economic Behavior 64, 2 (2008), 612-631.

[9] Weijie Shi, Linquan Zhang, Chuan Wu, Zongpeng Li, and Francis CM Lau. 2015 An online auction framework for dynamic resource provisioning in cloud computing. IEEE/ACM transactions on networking 24, 4 (2015), 2060-2073.
[10] Kasthuri Srinivasan and Satoshi Fujita. 2014. Truthful allocation of virtual machine instances with the notion of combinatorial auction. In 2014 Second International Symposium on Computing and Networking. IEEE, 586-590.

[11] Hong Zhang, Hongbo Jiang, Bo Li, Fangming Liu, Athanasios V Vasilakos, and Jiangchuan Liu. 2016. A framework for truthful online auctions in cloud computing with heterogeneous user demands. IEEE Trans. Comput. 65, 3 (2016), 805-818.

[12] Linquan Zhang, Zongpeng Li, and Chuan Wu. 2014. Dynamic resource provisioning in cloud computing: A randomized auction approach. In IEEE Infocom Proceedings. IEEE Computer Society. The Journal's web site is located at http ...

[13] Qi Zhang, Quanyan Zhu, and Raouf Boutaba. 2011. Dynamic resource allocation for spot markets in cloud computing environments. In 2011 Fourth IEEE International Conference on Utility and Cloud Computing. IEEE, 178-185.

[14] Xiaoxi Zhang, Zhiyi Huang, Chuan Wu, Zongpeng Li, and Francis CM Lau. 2016. Online auctions in IaaS clouds: Welfare and profit maximization with server costs. IEEE/ACM Transactions On Networking 25, 2 (2016), 1034-1047. 